\title{
FATAL NEPHRITIS COMPLICATING IDIOPATHIC PULMONARY HAEMOSIDEROSIS IN YOUNG ADULTS
}

BY

\author{
CATHERINE S. MACGREGOR, R. SLEIGH JOHNSON, AND K. A. D. TURK \\ From the Chelmsford Group of Hospitals
}

(RECEIVED FOR PUBLICATION JANUARY 28, 1960)

While many cases of idiopathic pulmonary haemosiderosis have been recorded in recent years, predominantly in children, the disease is rare in adolescents and adults, and is still of unexplained aetiology. Hitherto, the occurrence of any form of renal disease has been mentioned in only a few case reports. Attention has recently been drawn to an association between haemorrhagic pulmonary disease and severe glomerular disease of the kidney by the papers of Parkin, Rusted, Burchell, and Edwards (1955) and Heptinstall and Salmon (1959). Five of the 10 cases described in these two papers were found at necropsy to have polyarteritis nodosa. Two others, described by Heptinstall and Salmon, had changes in the lungs typical of idiopathic pulmonary haemosiderosis. This paper describes two young adults whose illness conformed to the accepted clinical and pathological pattern of idiopathic pulmonary haemosiderosis, but was complicated by fulminating fatal nephritis.

\section{Case Reports}

CASE 1.-A boy, aged 16 years 10 months, was admitted on March 3, 1956, with haemoptysis. A mild degree of breathlessness without cough had followed pneumonia at the age of 1 . For three to four months he had increasingly severe dyspnoea and he had frequently coughed up about a teaspoonful of bright blood, with as much as a cupful a day before admission. Little affection of other systems was present, and his weight and temperature had remained normal. No tuberculosis contact was known, nor any previous history given of rheumatism, nephritis, or other serious illness.

On admission he had a raised pulse rate and moderate pallor, but no distress. A few basal crepitations were present, the abdomen, skin, and lymph nodes showing no abnormality, while for five weeks he was afebrile.

A blood count then showed a hypochromic anaemia with $57 \%$ haemoglobin $(8.4$ g.) and $3,340,000$ R.B.C.s per c.mm., without specific features or abnormality of leucocytes, the serum bilirubin being $0.16 \mathrm{mg}$. per $100 \mathrm{ml}$. Treatment for a month with oral ferrous sulphate 6 grains $(390 \mathrm{mg}$.) t.d.s. was followed by a rise of haemoglobin only to $66 \%(9.7 \mathrm{~g}$.), the leucocytes remaining normal and reticulocytes being $2.8 \%$.
Brisk haemoptysis continued for a month as the one striking symptom. Examination of the mouth and upper respiratory tract showed no source of bleeding, nor was any evidence found of such possible causes of pulmonary haemorrhage as tuberculosis, bronchopneumonia, or primary blood disease. Radiographs of the chest on March 5 showed an extensive, soft, illdefined mottling throughout both lung fields, most marked in the lower lobes where the areas of consolidation were dense and confluent. No cavitation or pleural reaction was evident, the hilar shadows were slightly prominent, and the heart shadow appeared normal. These changes were noted to coincide clinically with a severe bout of haemoptysis. Repeated search of sputum for tubercle bacilli was negative, and the general bac teriology uninformative. The Mantoux reaction was negative in dilutions of 1 in 10,000 and 1 in 1,000. The severity of the haemoptysis was uninfluenced by 10-day course of intramuscular penicillin, given for $\vec{a}$ possible pneumonic pathology, and the finding on one occasion of sensitive streptococci.

Possible alternative diagnoses of sarcoidosis and polyarteritis nodosa were considered, but no supporting biochemical or radiological evidence was found. The urine was of 1.020 specific gravity and free from albumin, sugar, blood, and bile. Bronchoscopy on April 6 under local anaesthesia failed to reveal any lesion in the larynx, trachea, or visible bronchial subdivisions which would explain the source of pulmonary bleeding.

After a few days the haemoptysis temporarily ceased, and successive chest films showed absorption of pulmonary opacities in all areas, with complete clearing two weeks later. There was corresponding good clinical progress, so the patient was discharged home on April 13 for out-patient review, with a tentative diagnosis of atypical pneumonia. Haemoptysis of smaller degree continued, however, without other complaint or incapacity, the clinical and radiological chest findings remaining normal. The haemoglobin rose to $85 \%$ (12.6 g.), and no other abnormality in the blood was then present. Examination by bronchography was intended at a more suitable stage to exclude bronchiectasis as a possible source of bleeding.

Three months later a marked deterioration occurred, leading to his readmission on August 10. About half an ounce of bright or dark blood was coughed up many times daily without accompanying sputum: a few skin 
petechiae were present, and there was malaise. The blood count fell to $34 \%$ haemoglobin and 1,790,000 R.B.C. per c.mm., with no other abnormal findings except for a raised sedimentation rate (Westergren) of $75 \mathrm{~mm}$. in one hour and a reticulocyte count of $2 \%$. A biopsy of voluntary muscle showed no vascular abnormality suggestive of polyarteritis nodosa. Chest radiographs showed extensive fine mottled consolidation throughout both lung fields, which then appeared compatible with a diagnosis of idiopathic pulmonary haemosiderosis.

An unexpected and disturbing development, however, was the onset of an acute nephritis, shown by facial oedema, rise of blood pressure to $160 / 80 \mathrm{~mm}$. $\mathrm{Hg}$, without fundal changes, and a progressive reduction in urinary output to about $6 \mathrm{oz}$. $(170 \mathrm{ml}$.) a day. There was a heavy cloud of albumin with moderate numbers of red cells and a few leucocytes in the deposit, but the culture was sterile. The blood-urea level, $240 \mathrm{mg}$. per $100 \mathrm{ml}$. a week after admission, rose rapidly to $393 \mathrm{mg}$. per $100 \mathrm{ml}$. three days later, with a relative increase in the serum globulin.

A Bull's régime of diet was instituted, and because of the severe, resistant anaemia a slow transfusion was given of 3 pints of compatible whole blood, without reaction. The nephritis pursued a rapid and severe course, with mental confusion, drowsiness, oliguria, and frequent vomiting, so that all fluids had to be given intravenously. The serum potassium rose to $31 \mathrm{mg}$. per $100 \mathrm{ml}$. (7.9 mEq. per litre), with reduced alkali reserve and characteristic E.C.G. changes. Cortisone, $50 \mathrm{mg}$., was given six hourly for 10 days, together with penicillin and tetracycline, oral " resonium A" and an intracaval infusion of $40 \%$ dextrose solution, but his condition deteriorated rapidly. He developed intractable vomiting, complete anuria, and died on August 31, three weeks after readmission.

Post-mortem Findings.-Poth lungs were firmer and heavier than normal, with their pleural surfaces vividly mottled purple and brown. The cut surfaces of all lobes had a uniform, rusty-brown appearance with many irregular, ill-defined, reddish areas. The air passages contained blood-stained fluid. All the bronchial lymph nodes were reddish-brown and enlarged. No obvious bleeding point could be found in the lungs.

The kidneys were normal in size, the right weighing $150 \mathrm{~g}$. and the left $165 \mathrm{~g}$. The cortices showed loss of pattern, with many petechial haemorrhages.

The pancreas was enlarged and firm and the ducts contained opaque, mucoid material. Near the ampulla of Vater, the main pancreatic duct was blocked by inspissated secretion. No significant abnormality was found in any other organ. Permission to examine the head was not obtained.

Microscopic Changes.-Blocks from representative parts of each lobe of the lungs were examined; all were found to show similar changes. Only about $20 \%$ of the alveoli contained air, and these were greatly distended. The rest showed evidence of old or recent intra-alveolar haemorrhage, being filled by varying proportions of red blood cells, haemosiderin-laden macrophages, and free granules of haemosiderin. In areas of older haemorrhage some alveolar walls were thickened, owing partly to swelling of the lining cells and partly to increase of reticulin and collagen fibres. A few haemosiderin-laden macrophages were present in the walls at these points. Stains for elastic tissue showed mild fragmentation of the fibres in areas of old haemorrhage but no abnormality elsewhere. About $5 \%$ of the alveoli showed evidence of fresh haemorrhage, being filled with red cells and fibrin only. The walls of these alveoli appeared normal. The impression was gained that thickening of alveolar walls was proportional to the age of the haemorrhage in any given area of the lung, and therefore represented a gradually developing reaction to the presence of blood, or its breakdown products, in the alveoli. None of the alveoli showed organization of the contained blood clot, and a variety of stains failed to reveal any necrosis of alveolar walls.

The bronchi and the pulmonary and bronchial vessels were normal. No evidence of arteritis could be found. The sinuses of the bronchial lymph nodes were full of haemosiderin-laden macrophages.

The kidneys showed severe, diffuse, necrotizing glomerulo-nephritis. Most glomeruli were greatly enlarged, over-cellular, and avascular owing to proliferation of capillary endothelial cells. Areas of " fibrinoid" necrosis of varying sizes were present in many glomerular tufts; these contained a few recognizable red cells, and gave positive reactions for fibrin with the picro-Mallory stain. All glomerular tufts showed multiple capsular adhesions, and in many cases the capsular space was completely obliterated. Where the space persisted, it often contained red blood cells and showed proliferation of the lining epithelium, forming early crescents. In about half the glomeruli there was some degree of fibroblastic proliferation and early fibrosis of the tuft, with corresponding pericapsular fibrosis. About $10 \%$ of the glomeruli were small, hyalinized and fibrotic. No normal glomeruli were found. All the cortical tubular epithelium showed mild hyaline droplet degeneration, but there did not appear to be any actual necrosis of the epithelium. The renal vessels appeared normal.

Sections of the pancreas showed dilatation of the ducts, which contained amorphous eosinophilic material and many polymorphs. Polymorphs had heavily and diffusely infiltrated the parenchyma and interstitial tissue. Areas of recent fat necrosis were present in the adipose tissue around the pancreas.

Other organs did not show any significant histological abnormality. There was no evidence of acute or healed arteritis.

CASE 2.-A youth aged 19 years was admitted on January 28,1958 , with suspected pulmonary tuberculosis. His past health had been good, with no rheumatism or other relevant illness, nor any known tuberculosis contact. When he had coughed up a few specks of brownish blood six months earlier, his chest radiograph was normal, but he had since continued to expectorate small clots unaccompanied by sputum, though he was not otherwise ill. A month before admission he had developed cough and increasing dyspnoea on exertion, 


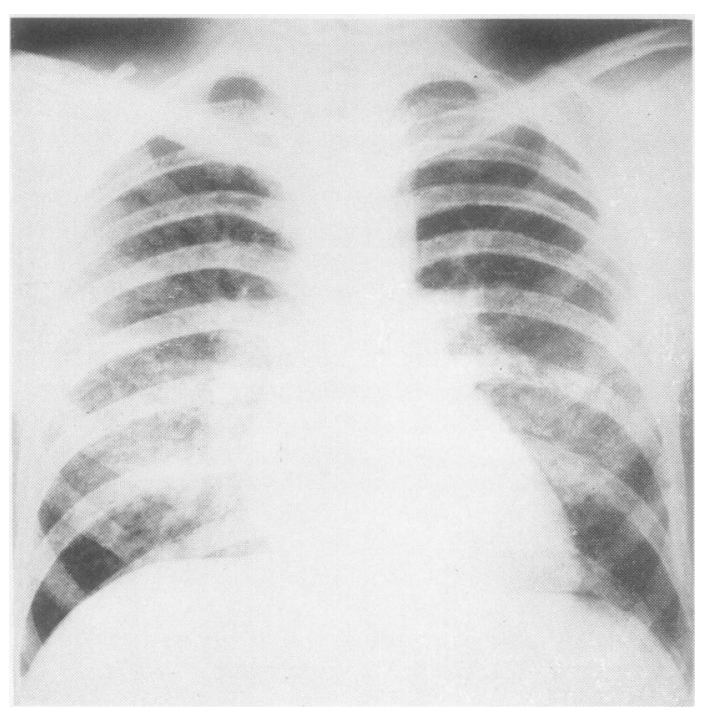

Fig. 1.-Case 2: Postero-anterior film of chest on January 28, showing diffuse soft focal ground-glass consolidation of both lung fields, most marked in the perihilar areas. with marked fatigue and malaise. This time the chest radiographs resembled miliary tuberculosis, and led to his admission.

He then had pallor of the mucous membranes but no dyspnoea at rest or congestive changes; physical examination of the chest, abdomen, and skin was negative, and the blood pressure was $120 / 60 \mathrm{~mm}$. $\mathrm{Hg}$. The chest radiograph (Fig. 1) showed a fine "ground glass" reticulation throughout the lung fields, more marked in both mid zones. Repeated search for tubercle bacilli was negative, and no other pathogenic organisms were found in the sputum; the Mantoux reaction was negative in a 1 in 1,000 dilution.

Examination of the blood showed a normochromic, normocytic anaemia, with $32 \%$ haemoglobin $(4.7 \mathrm{~g}$. $\mathrm{Hb})$, and 1,700,000 R.B.C.s per c.mm. A differential leucocyte count was normal, as were the bleeding and coagulation times and prothrombin index. The urine contained a heavy cloud of albumin and numerous red blood cells.

The anaemia was treated on the evening of admission by a slow transfusion of six bottles (3.2 litres) of compatible blood, with a subsequent rise in haemoglobin content to $74 \%(10 \mathrm{~g}$. $\mathrm{Hb})$ over the next few days. Examination of the urine on the day after admission showed that, despite a good output, blood was present in an estimated proportion of $5 \mathrm{ml}$. per litre of urine

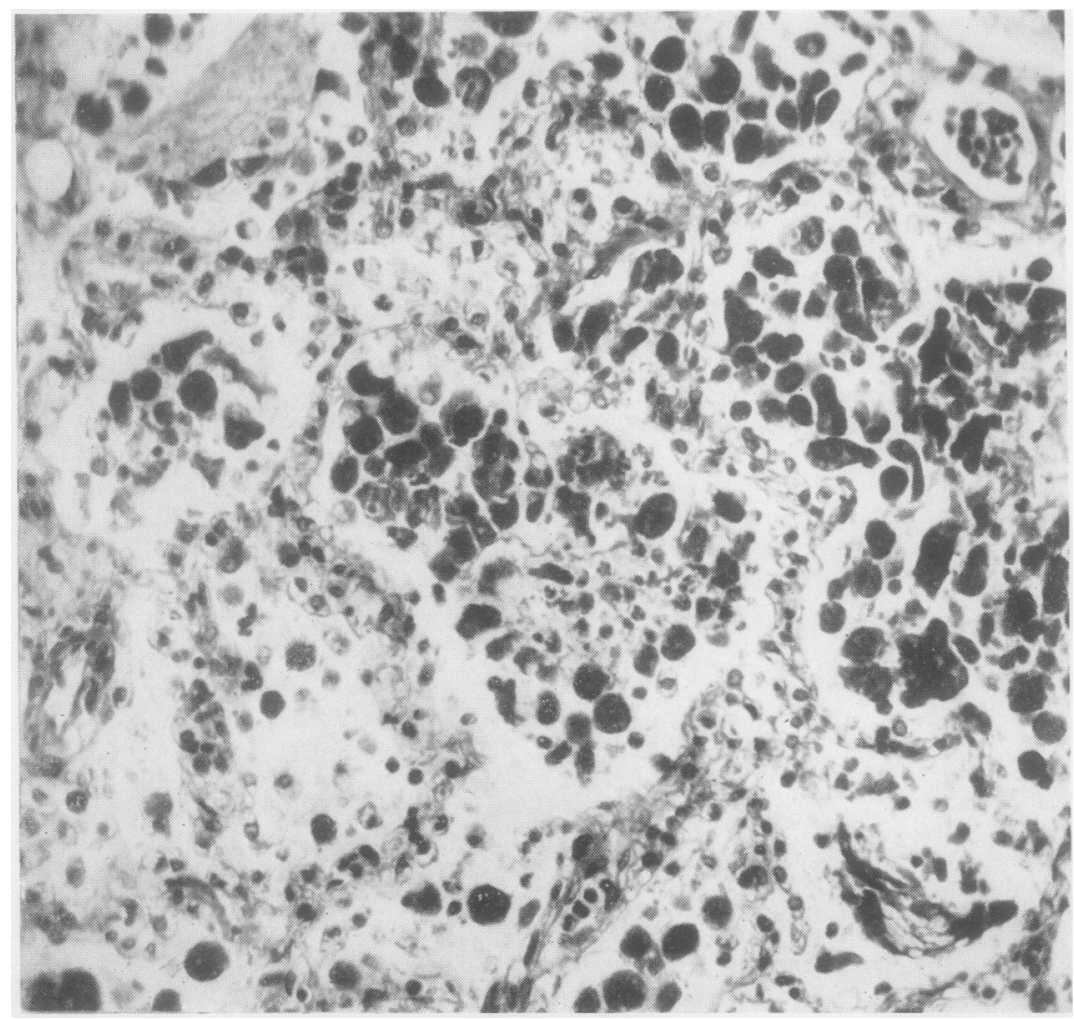

FIG. 2.-Case 1: Lung with haemosiderin-laden macrophages in alveoli. Picro-Mallory $\times 280$. 
passed, with occasional granular casts, but several urine cultures were sterile. The blood urea on admission was $276 \mathrm{mg}$. per $100 \mathrm{ml}$. Tests of the faeces for occult blood were negative, and liver function tests, made because of possible polyarteritis nodosa, were normal. A biopsy of voluntary muscle was not attempted because of the patient's weak condition. Serological reactions for Weil's agglutinins were also negative.

Treatment of $25 \mathrm{mg}$. oral "prednisone" was given daily for the nephritis. We gave streptomycin and isoniazid at first in view of a possible tuberculous aetiology of the pulmonary lesions, but when it was obvious that the renal condition was worsening the streptomycin was withdrawn after a total of $2 \mathrm{~g}$. had been given.

Some initial clinical improvement followed the transfusion, but the chest radiograph showed little change, except for further increase in prominence of mid-zone markings. The patient continued to expectorate small dark blood clots, without accompanying sputum. The customary measures of a Bull's régime were instituted early, but although we increased the "prednisone" and later gave intravenous cortisone, we could not stop the advancing nephritis. The urine output diminished progressively, with a rapid rise of the blood urea to $600 \mathrm{mg}$. per $100 \mathrm{ml}$. and of the serum potassium to
$38.2 \mathrm{mg}$. per $100 \mathrm{ml}$. (9.8 mEq. per litre). The clinical picture became one of uraemia with intractable retching, vomiting, and irritability, ending in death on the eleventh day after admission.

Post-mortem Findings.-Striking changes were found in the lungs and kidneys, which immediately recalled the first patient. The lungs were grossly oedematous, bulky and firm, and showed the same uniform rusty-brown colour, though this was less marked than in Case 1. The bronchi were full of frothy, blood-stained fluid. The bronchial lymph nodes were brown, but not enlarged.

The right kidney weighed $170 \mathrm{~g}$. and the left $176 \mathrm{~g}$. The cut surfaces showed swollen, patternless cortices which were dark red and dripped blood.

The other organs were not remarkable.

Microscopic Changes.-Sections from all lobes of the lungs showed changes essentially similar to those seen in Case 1. The degree of haemosiderosis in all areas was considerably less, but there was more evidence of recent haemorrhage. The alveolar walls in some areas of older haemorrhage showed moderate thickening. Organization of the contained blood clot had occurred in a few alveoli. There was no evidence of necrosis of alveolar walls or of vasculitis. The elastic tissue appeared normal in all areas. A number of small venules near areas of older
Fig. 3.-Case 1: Kidney with a glomerulus showing cellular proliferation and fibrinoid necrosis. Nearby is another glomerulus showing fibroblastic proliferation and early hyalinization. Haematoxylin and eosin $\times 120$.

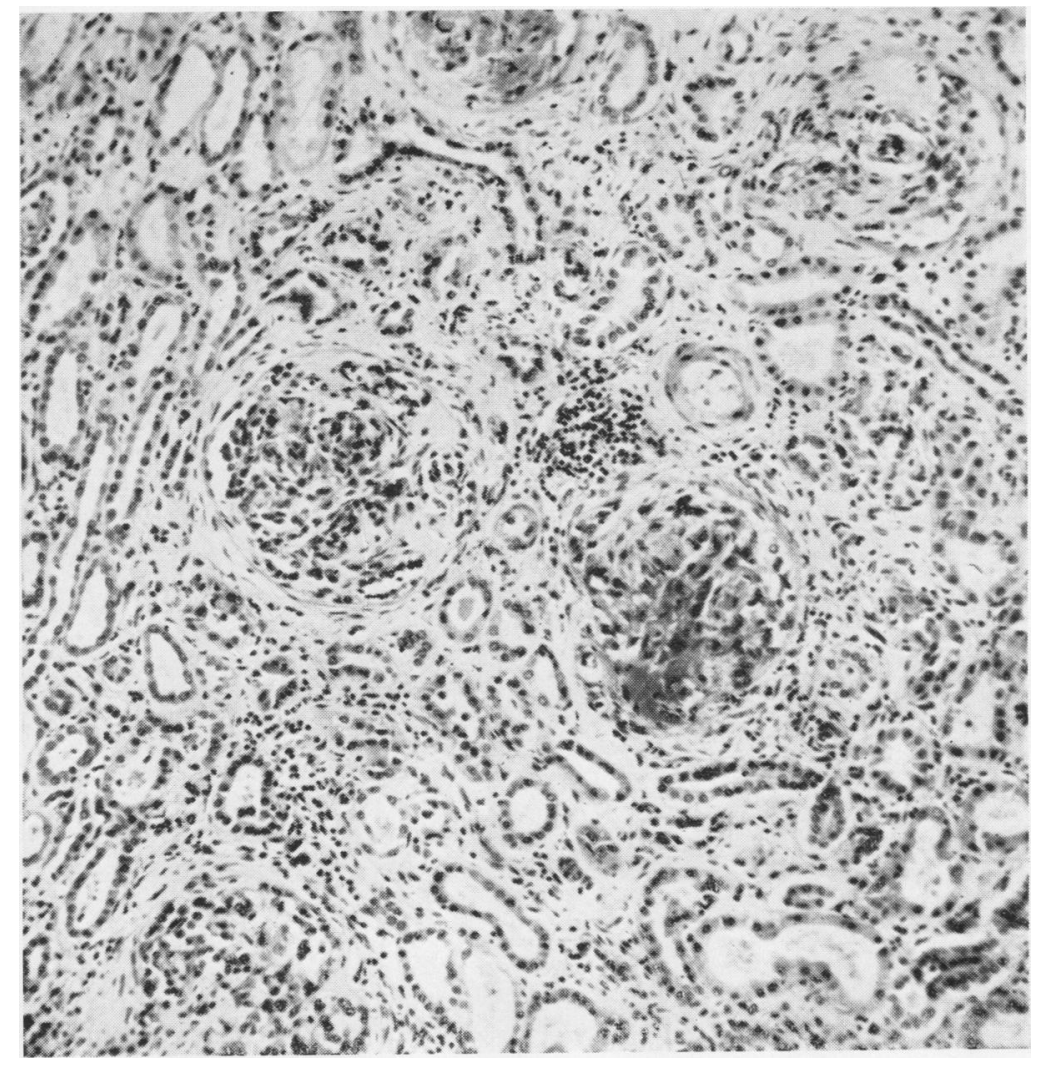


haemorrhage showed diffuse staining of their walls by haematoxylin; the prussian blue reaction demonstrated that the vessel walls at these points were diffusely impregnated with free iron.

The kidneys showed a diffuse necrotizing glomerulonephritis almost identical with the first patient. No normal glomeruli were found, all showing proliferative glomerulitis with areas of fibrinoid necrosis. Fibroblastic proliferation in the glomerular tufts was generally less marked than in Case 1, and no completely sclerosed glomeruli were seen. The renal vessels were normal.

Sections of other organs showed severe congestive changes only. There was no evidence of arteritis in any organ.

\section{Discussion}

Clinically and pathologically these two patients showed strikingly similar features. Both suffered from repeated haemoptyses, severe anaemia, and dyspnoea, the first for 10 months and the second for six months before death. Both developed a rapidly progressive, fatal nephritis approximately one month before death. Neither had a significant rise in blood pressure. At necropsy the lungs of both showed evidence of widespread old and recent intra-alveolar haemorrhage. The first patient, in whom the haemoptysis had been more severe and of longer duration, showed a greater degree of haemosiderosis. Neither had any primary heart disease or localized bleeding point in the lungs. Necrotizing alveolitis was not present. The changes in the lungs, and the clinical picture up to the time of onset of nephritis, conformed to the accepted pattern of idiopathic pulmonary haemosiderosis.

The renal changes were very similar. All glomeruli were abnormal, necroses being present in some, while most showed proliferation of the cells of the tufts and of the capsular epithelium, with crescent formation. Some partly sclerosed glomeruli were found in the first but not in the second. The histological picture of the kidneys in both was that of rapidly progressive Type 1 nephritis (Ellis, 1942), or extracapillary type of subacute glomerulonephritis (Volhard and Fahr, 1914), although more necroses were present in the glomerular tufts than is usual in this type of nephritis.

We did not know why a mild acute pancreatitis was found in the first patient, although similar changes have been attributed to cortisone therapy (Carone and Liebow, 1957).

Idiopathic pulmonary haemosiderosis is predominantly a disease of childhood, but increasing examples are now being recognized in adults. By 1955 Wynn-Williams and Young were able to find 15 published cases, ranging in age from 15 to 38 years at the time of diagnosis (Wynn-Williams and Young, 1956). Heptinstall and Salmon (1959) have recently reviewed the published cases occurring at $\overrightarrow{\vec{F}}$ all ages, to see if there is a high incidence of renal lesions. Out of a total of 69 they found four in whom a renal lesion was present, described by the authors as focal embolic nephritis, focal nephritis, focal acute to subacute glomerulo-nephritis and focal acute glomerulo-nephritis, respectively. A is fifth patient appears to have had polyarteritis $\vec{\circ}$ nodosa.

Parkin et al. (1955) described seven patients with fatal glomerulo-nephritis, in whom haemoptysis was a prominent feature. At necropsy all showed " acute necrotizing alveolitis which bore similarity to pulmonary lesions described previously in hypersensitive animals and humans." Four of their patients had clear evidence of polyarteritis nodosa in the spleen and other organs, but no arteritis could be found in the other three; it appears probable that these do not form a homogeneous group. Necrotizing alveolitis was not present in our cases. Scadding (1958, Case 4) described a case as being possibly related to those of Parkin et al. His patient was a man aged 29 years who had recurrent haemoptysis, anaemia, and slight cough for nine months before the onset of progressive renal failure, from which he died two months later. A lung biopsy two month before death showed changes similar to those of idiopathic pulmonary haemosiderosis and necropsy there was severe glomerulo-nephritis with hyalinization and destruction of many glomeruli. Scadding (personal communication) could find no clear evidence of necrotizing alveolitis, and there was no evidence of polyarteritis, so that his patient appears to be essentially similar to ours and distinct from those of Parkin et al.

Heptinstall and Salmon (1959) described three patients suffering from pulmonary haemorrhage and extensive glomerular disease of the kidney. Their first had polyarteritis, and will not be further considered here. Their second was a man of 23 years in whom recurrent haemoptysis was followed within two months by dyspnoea with gross anaemia, and a progressive nephritis from which death occurred about four weeks later. In this patient the same type of mid-zone pulmonary opacities and coarse miliary mottling of the lung fields were present as in our patients, and miliary tuberculosis was suspected. At necropsy there was haemosiderosis of the lungs, and an acute glomerulo-nephritis affected all the glomeruli, with crescent formation, some lesions resembling fibrinoid necroses. There was no evidence of polyarteritis or necrotizing alveolitis.

Their third patient was a man aged 41 years with a three months' history of intermittent haemoptysis 
and possibly haematuria. He had a severe anaemia, a blood urea level of $180 \mathrm{mg}$. per $100 \mathrm{ml}$., and developed heart failure, and pulmonary oedema, hypertension, and retinal haemorrhages. Prednisone treatment produced no improvement apart from subsidence of the haemoptysis. Dialysis reduced the blood urea from 290 to $80 \mathrm{mg}$. per $100 \mathrm{ml}$., with clinical improvement for 11 days. A second dialysis was performed, but death occurred the next day. Necropsy showed marked pulmonary haemosiderosis and a diffuse glomerulo-nephritis, with many completely hyalinized glomeruli. There was no polyarteritis or necrotizing alveolitis. Heptinstali and Salmon considered that the pulmonary changes in their second and third patients were those of idiopathic pulmonary haemosiderosis. The renai changes in their second patient were accepted as the extracapillary type of subacute glomerulo-nephritis (Volhard and Fahr, 1914), but they commented on the unusual prominence of necroses in the tufts. The renal changes in their third patient seemed a more chronic glomerulo-nephritis with recent proliferative activity.

Patients 2 and 3 of Heptinstall and Salmon (1959), patient 4 of Scadding (1958), and the present two form a strikingly homogeneous clinical and pathological group; it may be of significance that these five were all males. It appears that young adults suffering from idiopathic pulmonary haemosiderosis are liable to develop a rapidly progressive form of diffuse glomerulo-nephritis, characterized histologically by unusually numerous necroses in the glomerular tufts. Where a dramatic nephritis ensues, attention is liable to be focused on the renal disease, especially if the pulmonary symptoms and the post-mortem changes in the lungs are not severe. Also, confusion with polyarteritis, Wegener's granulomatosis (1939), and other diseases affecting the lungs as well as the kidneys is liable to occur. We suspect that some patients with idiopathic pulmonary haemosiderosis, complicated by nephritis, are not recognized, and that the syndrome may not be very uncommon.

Treatment of idiopathic pulmonary haemosiderosis, other than by symptomatic measures, has not been very effective. Browning and Houghton
(1956) record one recovery following A.C.T.H. and cortisone therapy. The third patient of Heptinstall and Salmon (1959) was treated with prednisone, and although haemoptysis ceased after two days, the course of the nephritis was not altered. Both our patients were given steroids, without obvious benefit, and we think that even if steroid therapy can arrest the pulmonary haemorrhage, it is unlikely to affect the downhill course of the patient once nephritis has developed.

The natural history and morbid anatomy of idiopathic pulmonary haemosiderosis suggest that the essential lesion is in the pulmonary capillaries, leading to recurrent leakage of blood into the alveoli. Acute diffuse glomerulo-nephritis of extracapillary type has long been thought to be an inflammatory capillaritis affecting the glomerular tufts, with leakage of blood into the capsular spaces. There may be a common lesion in the capillaries of the lungs and kidneys in our cases, though the nature of such a lesion remains obscure. An explanation on the basis of hypersensitivity is attractive, but as yet purely speculative.

\section{SUMMARY}

Two young men with idiopathic pulmonary haemosiderosis are described, each of whom developed a rapidly fatal, diffuse glomerulo-nephritis of extracapillary type, with unusually numerous "fibrinoid" necroses in the glomerular tufts. Three previously recorded cases, showing close similarities, are discussed and compared. There is no evidence that the syndrome is a manifestation of polyarteritis ; the most likely basis of the condition is a diffuse capillary lesion in the alveolar walls of the lungs and in the glomerular tufts.

\section{REFERENCES}

Browning, J. R., and Houghton, J. D. (1956). Amer. J. Med., 20, 374. Carone, F. A., and Liebow, A. A. (1957). New Engl. J. Med., 257 690

Ellis, A. W. M. (1942). Lancet, 1, 1.

Heptinstall, R. H., and Salmon, M. V. (1959). J. clin. Path., 12, 272. Parkin, T. W., Rusted, I. E., Burchell, H. B., and Edwards, J. E. (1955). Amer. J. Med., 18, 220.

Scadding, J. G. (1958). Proc. roy. Soc. Med., 51, 653

Volhard, F., and Fahr, T. (1914). Die Brightsche Nierenkrankheit. Springer, Berlin.

Wegener, F. (1939). Beitr. path. Anat., 102, 36

Wynn-Williams, N., and Young, R. D. (1956). Thorax, 11, 101. 